\title{
Effet de la nature de la jachère sur la colonisation de la culture subséquente par les champignons endomycorhiziens : cas du système 'jachère' manioc sur sols ferrugineux tropicaux du Bénin
}

\author{
Aliou SAIDOU ${ }^{1^{*}}$, Dansou KOSSOU ${ }^{1}$, Anastase AZONTONDE ${ }^{2}$ et \\ Déo-Gratias J. M. HOUGNI ${ }^{1}$ \\ ${ }^{1}$ Département de Production Végétale, Faculté des Sciences Agronomiques, \\ Université d'Abomey Calavi 01 BP 526 RP Cotonou Bénin. \\ ${ }^{2}$ Laboratoire des Sciences du Sol, Eau et Environnement (LSSE), Centre de Recherche d'Agonkanmey, \\ Institut National des Recherches Agricoles du Bénin (INRAB), 01 BP 988 Cotonou Bénin. \\ *Auteur correspondant, E-mail: saidoualiou@ gmail.com, Tél : (+229) 974944 80, Fax.: (+229) 21303084
}

\section{RESUME}

En vue d'évaluer les arrières effets d'une fumure d'engrais minéral sur la croissance du maïs et la capacité des champignons endomycorhiziens à mobiliser le phosphore, quatre cultures successives de maïs ont été conduites en pots sous serre sur des échantillons de sol collectés au niveau de trois types de 'jachère' manioc ayant comporté les variétés de manioc Ben 86052, Bouaké (variété traditionnelle introduite), et odongbo (variété locale utilisée comme référence). Deux doses d'engrais complexe $\mathrm{N}_{14} \mathrm{P}_{23} \mathrm{~K}_{14}(0$ et $100 \mathrm{~kg} / \mathrm{ha}$ correspondant à $14 \mathrm{~kg}$ de $\mathrm{N} ; 10,12 \mathrm{~kg}$ de $\mathrm{P}$ et $11,62 \mathrm{~kg}$ de $\mathrm{K}$ ) ont été appliquées à la première culture de maïs en champ en 2004. On note une baisse significative $(\mathrm{P}<0,001)$ des biomasses racinaires et aériennes du maïs en pot et de la quantité de $\mathrm{P}$ mobilisée par les plants au cours des cultures successives avec un effet plus prononcé au niveau de la 'jachère' Ben 86052. Les types de 'jachère', l'effet de la précédente fumure et la succession des cultures influent significativement $(\mathrm{P}<0,001)$ sur le taux de colonisation des racines par les spores des champignons endomycorhiziens. On note des corrélations hautement significatives $(\mathrm{P}<0,001)$ entre le taux de colonisation des racines par les spores des champignons endomycorhiziens, et la quantité de $\mathrm{P}$ mobilisée. (C) 2009 International Formulae Group. All rights reserved.

Mots clés : champignons endomycorhiziens, jachère manioc, maïs, biomasse végétale, effet résiduel de fumure.

\section{INTRODUCTION}

La baisse de la fertilité des sols est une contrainte agricole majeure en Afrique subsaharienne (Sanchez et Jama, 2002; Erenstein, 2003). Dans les systèmes traditionnels de culture, la restauration de la fertilité des sols est souvent assurée par les jachères naturelles de longue durée (Serpantié et Ouattara, 2001; Kolawolé et al., 2003). De nos jours, la poussée démographique ayant pour conséquence l'augmentation des besoins alimentaires a accru les besoins en terres fertiles (Fermont et al., 2008). Cette situation se traduit par une réduction de la durée des périodes de jachère sans autre mesure visant la restitution des nutriments utilisés par les cultures (Saïdou, 2006; Fermont et al., 2008). L'appauvrissement des sols se trouve ainsi accéléré et devient de ce fait un problème d'envergure (Sanchez et Jama, 2002).

Face à son aggravation et au faible taux d'adoption (environ 7\%) (Douthwaite et al., 2002) des technologies proposées par les services de vulgarisation, les paysans développent des stratégies encore mal connues du monde scientifique. L'une d'elles est la 
'jachère' manioc (Saïdou et al., 2004; AdjeiNsiah et al., 2007; Fermont et al., 2008; Saïdou et al., 2008), que l'on rencontre au sud et au centre Bénin et un peu partout en Afrique de l'Ouest, et dont les effets sur la fertilité du sol opposent producteurs et scientifiques (Saïdou, 2006). Adjei-Nsiah et al. (2007) et Saïdou et al. (2008) expliquent les performances de ce système de culture par l'importance de la litière déposée par le manioc, l'intensification de l'activité biologique dans le sol en particulier des vers de terre contribuant au recyclage des nutriments dans le sol et surtout la biodisponibilité du phosphore due à l'activité mycorhizienne.

En effet, le manioc est facilement colonisé par les champignons endomycorhiziens (Habte et Byappanahalli, 1994; Howeler, 2002; Fermont et al., 2008). Ces derniers améliorent la production du manioc à travers l'amélioration de la mobilisation des nutriments (Fagbola et al., 1998; Cardoso et Kuyper, 2006). Les champignons endomycorhiziens sont universellement reconnus pour leur aptitude à rendre disponible le phosphore difficilement accessible aux plantes (Cardoso et Kuyper, 2006; Mathimaran et al., 2007). Cette conversion des pools de phosphore est d'autant plus intéressante que les sols tropicaux en sont généralement déficients (Kolawolé et al., 2003).

La présente étude vise à évaluer les arrières effets d'une fumure minérale appliquée sous la forme d'un engrais complexe $\left(\mathrm{N}_{14} \mathrm{P}_{23} \mathrm{~K}_{14}\right)$ sur la production en biomasse d'une culture de maïs et la capacité des champignons endomycorhiziens à mobiliser le phosphore. De façon spécifique, l'étude vise à:(1) étudier l'effet résiduel d'une fumure minérale sur le taux de colonisation des racines de maïs par les champignons endomycorhiziens, et (2) étudier l'évolution de la mobilisation du $\mathrm{P}$ et des rendements (biomasse aérienne et racinaire) des cultures successives de maïs en pots subséquentes à différents types de 'jachère' manioc.

La principale hypothèse qui a guidé cette étude est que la fertilisation phosphatée a un effet dépressif sur la colonisation d'une culture subséquente de maïs par les champignons endomycorhiziens.

\section{MATERIEL ET METHODES}

L'expérimentation a consisté en la conduite de 4 cultures successives de maïs en pots sous serre contenant chacun $3,5 \mathrm{~kg}$ de terre avec un volume du pot de 3 litres. Les sols ont été prélevés à $0-20 \mathrm{~cm}$ de profondeur les 22 et 23 février 2005 sur des parcelles expérimentales du Projet Convergence des Sciences dans le village de Ouoghi (commune de Savè située à $8^{\circ} 07^{\prime}$ Nord, $2^{\circ} 33^{\prime}$ Est, sur sols ferrugineux tropicaux et sous climat soudano-guinéen à régime pluviométrique unimodal). Ces parcelles paysannes ont porté une succession culturale manioc-maïs en 2004. Au total, 12 échantillons de sol ont été prélevés à raison de 4 échantillons par cultivar de manioc (Ben 86052, Bouaké et odongbo). Les prélèvements de sol ont été effectués sur ces parcelles paysannes en raison de l'implication de leur propriétaire dans le programme de recherche du projet. Ces derniers sont sensibilisés sur les objectifs de la recherche et ont démontré une entière disponibilité à collaborer dans le cadre des activités du projet. Les propriétés physicochimiques des sols utilisés dans les pots sont résumées dans le tableau 1.

$\mathrm{Au}$ cours de chaque culture, les vases de végétation sont semés 24 heures après humectation du sol à raison de 3 graines de maïs par vase. Le démarrage a été fait à 2 plants par pot 14 jours après semis. Les vases sont arrosés en fonction des besoins en eau des plants à raison de 200 à $300 \mathrm{ml}$ par pot, correspondant à environ $4 / 9^{\mathrm{e}}$ de la capacité au champ des sols. Après chaque récolte effectuée 30 jours après semis, une période de deux semaines est observée avant l'installation de la culture suivante. Cette pratique permet un assèchement total du sol. Les pots n'ont pas été troués par le bas afin d'éviter des pertes par percolation de spores de champignons endomycorhiziens.

Les pots ont été disposés suivant un dispositif de bloc complètement aléatoire avec huit répétitions. L'essai comprend deux facteurs : l'application antérieure d'une fumure minérale aux doses de 0 et $100 \mathrm{~kg} / \mathrm{ha}$ du complexe $\mathrm{N}_{14} \mathrm{P}_{23} \mathrm{~K}_{14} \mathrm{~S}_{5} \mathrm{~B}_{1}$ (soient $14 \mathrm{~kg} / \mathrm{ha}$ de $\mathrm{N} ; 10,12 \mathrm{~kg} / \mathrm{ha}$ de $\mathrm{P}$ et $11,62 \mathrm{~kg} / \mathrm{ha}$ de $\mathrm{K}$ ) et trois types de 'jachères' manioc (cultivar Ben 86052 provenant de l'IITA introduit dans le village par les services de vulgarisation agricole, cultivar local Bouaké introduit dans 
Tableau 1: Propriétés physico-chimiques ( \pm erreurs standards) des sols ferrugineux tropicaux du village de Ouoghi au Centre Bénin utilisés dans le cadre de l'étude avant le démarrage des essais en pots en 2004.

\begin{tabular}{lccccc}
\hline Types de 'jachère' manioc & $\mathbf{p H}(\mathbf{e a u})$ & $\begin{array}{c}\text { C-organique } \\
(\mathbf{g} / \mathbf{k g})\end{array}$ & $\begin{array}{c}\text { N-total } \\
(\mathbf{g} / \mathbf{k g})\end{array}$ & $\begin{array}{c}\text { P-Bray 1 } \\
(\mathbf{m g} / \mathbf{k g})\end{array}$ & $\begin{array}{c}\text { K-éch. } \\
(\mathbf{c m o l} / \mathbf{k g})\end{array}$ \\
\hline Parcelle non fumée & & & & & \\
\hline Ben 86052 & $6,36 \pm 0,06$ & $7,8 \pm 0,40$ & $0,41 \pm 0,03$ & $32,53 \pm 2,48$ & $0,19 \pm 0,02$ \\
Bouaké & $6,54 \pm 0,05$ & $8,23 \pm 0,93$ & $0,43 \pm 0,08$ & $35,72 \pm 3,46$ & $0,20 \pm 0,01$ \\
Odongbo & $6,70 \pm 0,0$ & $10,12 \pm 0,32$ & $0,48 \pm 0,03$ & $35,91 \pm 3,43$ & $0,26 \pm 0,00$ \\
\hline Parcelle fumée & & & & & \\
\hline Ben 86052 & $6,39 \pm 0,10$ & $7,35 \pm 0,64$ & $0,30 \pm 0,04$ & $20,20 \pm 0,83$ & $0,31 \pm 0,02$ \\
Bouaké & $6,68 \pm 0,11$ & $8,94 \pm 0,62$ & $0,27 \pm 0,06$ & $21,30 \pm 2,81$ & $0,12 \pm 0,03$ \\
Odongbo & $6,58 \pm 0,15$ & $10,24 \pm 0,70$ & $0,55 \pm 0,11$ & $32,76 \pm 7,80$ & $0,18 \pm 0,04$ \\
\hline
\end{tabular}

le village à partir de la Côte d'Ivoire à travers les relations inter-paysannes, enfin le cultivar traditionnel Odongbo largement cultivé dans le village).

\section{Collecte de données}

La récolte de la biomasse aérienne a consisté à sectionner les plants de maïs à l'aide d'un sécateur au ras du sol 30 jours après semis (JAS). Le poids frais est mesuré à l'aide d'une balance de précision 0,1 gramme. La biomasse est ensuite mise à l'étuve à $60^{\circ} \mathrm{C}$ jusqu'à poids constant (pendant 48 heures) pour la détermination de la matière sèche. Ces échantillons sont par la suite conservés pour l'analyse des teneurs en P. Les racines de maïs sont ensuite collectées, lavées abondamment à l'eau de robinet dans le tamis, puis pesées pour la détermination du poids frais. Un échantillon est conservé au froid (environ 5 ${ }^{\circ} \mathrm{C}$ ) au réfrigérateur pendant $72 \mathrm{~h}$ pour l'estimation du taux de colonisation par les spores de champignon endomycorhizien. Une fraction des racines (de poids connu) est mise à l'étuve à $60^{\circ} \mathrm{C}$ jusqu'à poids constant, pour l'évaluation de la teneur en matière sèche.

Les prélèvements de sol ont été effectués au début et à la fin de l'essai en pots pour l'estimation du nombre de spores (le besoin en $\mathrm{P}$ est surtout important vers l'anthèse c'est-à-dire vers le milieu du cycle ce qui devrait être en corrélation avec l'infection des racines). L'extraction et l'estimation des spores dans le sol ont été faites suivant la méthode décrite par Brundrett et al. (1996). Les spores ont été estimées dans des verres de pétri quadrillés (méthode de grille) (Giovanneti et Mosse, 1980) sous un stéréo-microscope (Stemi DRC Zeiss) au grossissement $\times 40$. Seules les spores apparemment actives c'est-à-dire celles présentant des noyaux ont été prises en compte. Le nombre de spores a été rapporté au poids (en gramme) de sol sec.

Les racines fines du maïs conservées ont été colorées suivant la technique décrite par Brundrett et al. (1996) dans une solution de stain (1 g de chlorazol-black dans un rapport 1:1:1 glycérol, acide lactique et eau distillée). La technique de comptage est celle décrite par Brundrett et al. (1996). Le comptage est fait à l'aide du stéréomicroscope Zeiss (grossissement x 40). Les résultats sont exprimés en pourcentage de longueur de racine colonisée.

\section{Analyse de laboratoire et détermination de la biomasse}

Les échantillons de plants de maïs préalablement séchés et broyés ont été analysés pour leur teneur en $\mathrm{P}$ total suivant la méthode Duval (calcination de l'échantillon dans un four à moufle à $550{ }^{\circ} \mathrm{C}$ pendant $4 \mathrm{~h}$ de temps). La quantité de $\mathrm{P}$ mobilisée dans la biomasse a été ensuite calculée.

\section{Analyse des données}

Le traitement statistique des données a été effectué avec le logiciel Statistical Analysis System (SAS) v. 8.0 qui a permis l'analyse de variances à trois facteurs (types de jachère, fumure, cultures) suivant le modèle hiérarchisé suivi du test de séparation des moyennes (suivant la méthode de Student Newman-Keuls). La séquence des cultures a été introduite en plus des deux facteurs 
précédemment énoncés pour l'analyse de variance. Pour normaliser la distribution, les valeurs des nombres de spores dans le sol ont été transformées en $\log _{10}$ (n) et les valeurs relativisées (taux de colonisation des racines par les spores des champignons endomycorhiziens) ont été transformées en 2Arcsin $\sqrt{n}$ (Dagnelie, 1998), n étant la valeur réelle. Des corrélations ont également été établies entre la densité de spores dans le sol et les taux de colonisation racinaire d'une part et entre le taux de colonisation racinaire et les paramètres de production du maïs d'autre part.

\section{RESULTATS}

Effets des types de 'jachères' manioc, de la succession des cultures et de l'arrière effet d'une fumure minérale sur la production de biomasse du maïs

$\mathrm{La}$ production de matière sèche aérienne a significativement $(\mathrm{P}<0,001)$ baissé d'une culture à l'autre (Figure 1). Au cours des $2^{\text {ème }}, 3^{\text {ème }}$ et $4^{\text {ème }}$ séquences de culture, on a enregistré des baisses plus prononcées de la production de biomasse (aérienne) de maïs au niveau de la 'jachère' manioc Ben 86052 comparativement à la production de biomasse dans les 'jachères' manioc Bouaké et Odongbo sous l'arrière-effet de la fumure. L'arrière effet de la fumure minérale n'a été significatif $(\mathrm{P}<0,05)$ qu'à la première culture. Le poids sec des racines a baissé considérablement de $80,3 \%$ entre les deux premières cultures, puis de $23,3 \%$ avant de se
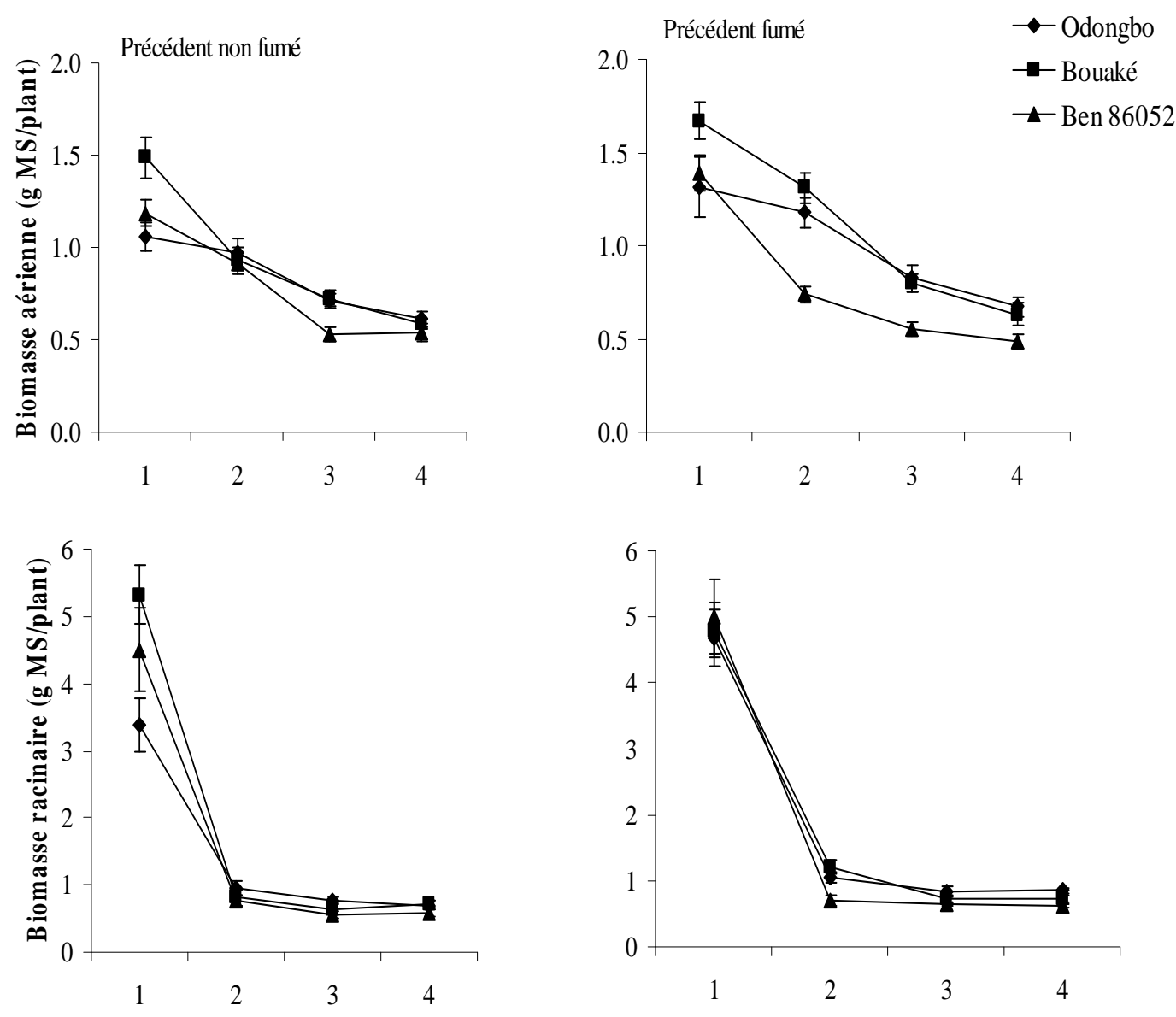

Succession des cultures

Figure 1: Effets des types de 'jachères' manioc, de l'arrière effet d'une fumure minérale $\mathrm{N}_{14} \mathrm{P}_{23} \mathrm{~K}_{14} \mathrm{~S}_{5} \mathrm{~B}_{1}$ et de la succession des cultures sur la production des biomasses (racinaire et aérienne) du maïs en pots. Les barres verticales représentent les erreurs standards. 
maintenir à la valeur de 0,70 g MS/plant pour les deux dernières cultures.

Effets des types de 'jachère' manioc, de la succession des cultures et des arrières effets d'une fumure minérale sur la concentration du $\mathbf{P}$ dans les tissus du maïs

Le tableau 2 présente la variation moyenne de la teneur en $\mathrm{P}$ des plants de maïs au cours des cultures successives sous l'influence des types de 'jachère' manioc et de l'effet résiduel d'une précédente fumure minérale. Entre la première et la deuxième culture, la teneur des plants en phosphore a significativement baissée $(\mathrm{P}<0,001$ et $\mathrm{P}<0,01$ respectivement pour les pots fumés et non fumés). Les différences entre les types de 'jachère' manioc d'une part et l'effet résiduel de la fumure minérale d'autre part se sont estompées dès la deuxième culture. Lors de la première culture, les teneurs en $\mathrm{P}$ enregistrées au niveau des types de 'jachère' manioc Odongbo sont significativement inférieures comparativement à celles des 'jachères' manioc Ben 86052 et Bouaké. De plus, les effets résiduels de la précédente fumure ont significativement $(\mathrm{P}<0,01)$ influencé la teneur des plants en $\mathrm{P}$. On note que les teneurs en $\mathrm{P}$ sont restées constantes de la troisième à la quatrième culture.

En ce qui concerne la mobilisation du $\mathrm{P}$ par les plants de maïs en pot, on note des différences hautement significatives $(\mathrm{P}<0,001)$ entre les types de 'jachère' manioc pendant les trois premières cultures (Tableau 3). L'effet résiduel de la précédente fumure minimale s'est révélé hautement significatif $(\mathrm{P}<0,001)$ pour la mobilisation du $\mathrm{P}$, mais uniquement pendant la $1^{\text {ère }}$ culture. Les plants cultivés sur sol provenant des 'jachères' manioc Bouaké mobilisent significativement $(\mathrm{P}<0,001)$ plus de $\mathrm{P}$ comparativement à ceux cultivés sur sol provenant de la 'jachère' Ben 86052 , et ceci lors des trois premières cultures.

Tableau 2: Valeurs moyennes ( \pm erreurs standards) de la concentration $(\mathrm{mg} / \mathrm{kg})$ des plants de maïs en $\mathrm{P}$ en fonction des types de 'jachère' manioc, de l'effet résiduel d'une précédente fumure minérale et des successions des cultures.

\begin{tabular}{|c|c|c|c|c|}
\hline $\begin{array}{l}\text { Succession des } \\
\text { cultures }\end{array}$ & Types de 'jachères' & Précédent non fumé & Précédent fumé & Moyennes \\
\hline \multirow{4}{*}{1} & Ben 86052 & $0,27 \pm 0,00 \mathrm{a}$ & $0,30 \pm 0,01 \mathrm{a}$ & $0,29 \pm 0,01 \mathrm{~A}$ \\
\hline & Bouaké & $0,29 \pm 0,01 \mathrm{a}$ & $0,29 \pm 0,01 \mathrm{ab}$ & $0,29 \pm 0,00 \mathrm{~A}$ \\
\hline & Odongbo & $0,24 \pm 0,01 \mathrm{~b}$ & $0,27 \pm 0,01 b$ & $0,25 \pm 0,01 \mathrm{~B}$ \\
\hline & Moyennes & $0,27 \pm 0,01 Y$ & $0,29 \pm 0,01 Y$ & \\
\hline \multirow{4}{*}{2} & Ben 86052 & $0,22 \pm 0,01 b$ & $0,26 \pm 0,01 \mathrm{a}$ & $0,24 \pm 0,01 \mathrm{~A}$ \\
\hline & Bouaké & $0,26 \pm 0,01 \mathrm{a}$ & $0,24 \pm 0,01 \mathrm{a}$ & $0,25 \pm 0,01 \mathrm{~A}$ \\
\hline & Odongbo & $0,27 \pm 0,00 \mathrm{a}$ & $0,24 \pm 0,01 \mathrm{a}$ & $0,25 \pm 0,01 \mathrm{~A}$ \\
\hline & Moyennes & $0,25 \pm 0,01 X$ & $0,25 \pm 0,00 X$ & \\
\hline \multirow{4}{*}{3} & Ben 86052 & $0,26 \pm 0,01 \mathrm{a}$ & $0,23 \pm 0,02 \mathrm{a}$ & $0,24 \pm 0,01 \mathrm{~A}$ \\
\hline & Bouaké & $0,25 \pm 0,01 \mathrm{a}$ & $0,24 \pm 0,01 \mathrm{a}$ & $0,25 \pm 0,01 \mathrm{~A}$ \\
\hline & Odongbo & $0,22 \pm 0,00 \mathrm{~b}$ & $0,24 \pm 0,01 \mathrm{a}$ & $0,23 \pm 0,01 \mathrm{~A}$ \\
\hline & Moyennes & $0,24 \pm 0,01 X$ & $0,24 \pm 0,01 X$ & \\
\hline \multirow{4}{*}{4} & Ben 86052 & $0,27 \pm 0,01 \mathrm{a}$ & $0,26 \pm 0,01 \mathrm{a}$ & $0,27 \pm 0,01 \mathrm{~A}$ \\
\hline & Bouaké & $0,26 \pm 0,01 \mathrm{a}$ & $0,25 \pm 0,01 \mathrm{a}$ & $0,26 \pm 0,01 \mathrm{~A}$ \\
\hline & Odongbo & $0,25 \pm 0,02 \mathrm{a}$ & $0,25 \pm 0,01 \mathrm{a}$ & $0,25 \pm 0,01 \mathrm{~A}$ \\
\hline & Moyennes & $0,26 \pm 0,01 X Y$ & $0,26 \pm 0,01 X$ & \\
\hline
\end{tabular}

N.B.: Les moyennes suivies de la même lettre alphabétique de même caractère et pour le même facteur (les lettres $a, b$ montrent les différences entre les jachères manioc considérant le facteur fertilisation et sans fertilisation; A, B montrent les différences entre les jachères manioc tous les niveaux de fertilisation confondus; $\mathrm{X}, \mathrm{Y}$ montrent les différences entre les niveaux de fertilisation tous les types de jachère manioc confondus) ne sont pas significativement différentes $(\mathrm{P}>0,05)$ d'après le test de Student Newman-Keuls. 
Tableau 3: Valeurs moyennes ( \pm erreurs standards) des quantités de P mobilisées dans la biomasse aérienne des plants de maïs en pot considérant les types de 'jachère' manioc, les résidus d'une précédente fumure minérale et la succession des cultures.

\begin{tabular}{|c|c|c|c|c|}
\hline $\begin{array}{l}\text { Succession } \\
\text { des cultures }\end{array}$ & Types de 'jachères' & $\begin{array}{l}\text { Précédent non fumé } \\
\text { (g/pot) }\end{array}$ & $\begin{array}{l}\text { Précédent fumé } \\
\text { (g/pot) }\end{array}$ & $\begin{array}{l}\text { Moyennes } \\
\text { (g/pot) }\end{array}$ \\
\hline \multirow[t]{4}{*}{ 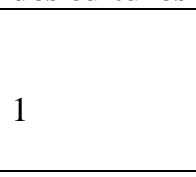 } & Ben 86052 & $0,32 \pm 0,02 b$ & $0,42 \pm 0,04 a$ & $0,37 \pm 0,03 \mathrm{~B}$ \\
\hline & Bouaké & $0,42 \pm 0,03 \mathrm{a}$ & $0,48 \pm 0,03 \mathrm{a}$ & $0,45 \pm 0,02 \mathrm{~A}$ \\
\hline & Odongbo & $0,26 \pm 0,03 b$ & $0,35 \pm 0,05 \mathrm{a}$ & $0,31 \pm 0,03 \mathrm{~B}$ \\
\hline & Moyennes & $0,3 \pm 0,02 Y$ & $0,42 \pm 0,03 X$ & \\
\hline \multirow{4}{*}{2} & Ben 86052 & $0,21 \pm 0,01 \mathrm{a}$ & $0,18 \pm 0,01 b$ & $0,19 \pm 0,01 \mathrm{~B}$ \\
\hline & Bouaké & $0,22 \pm 0,02 \mathrm{a}$ & $0,28 \pm 0,02 \mathrm{a}$ & $0,25 \pm 0,01 \mathrm{~A}$ \\
\hline & Odongbo & $0,26 \pm 0,03 a$ & $0,29 \pm 0,02 \mathrm{a}$ & $0,27 \pm 0,02 \mathrm{~A}$ \\
\hline & Moyennes & $0,23 \pm 0,01 X$ & $0,25 \pm 0,01 X$ & \\
\hline \multirow{4}{*}{3} & Ben 86052 & $0,14 \pm 0,01 b$ & $0,12 \pm 0,01 \mathrm{~b}$ & $0,13 \pm 0,01 \mathrm{~B}$ \\
\hline & Bouaké & $0,18 \pm 0,01 \mathrm{a}$ & $0,19 \pm 0,01 \mathrm{a}$ & $0,19 \pm 0,01 \mathrm{~A}$ \\
\hline & Odongbo & $0,16 \pm 0,01 \mathrm{ab}$ & $0,20 \pm 0,01 \mathrm{a}$ & $0,18 \pm 0,01 \mathrm{~A}$ \\
\hline & Moyennes & $0,16 \pm 0,01 X$ & $0,17 \pm 0,01 X$ & \\
\hline \multirow{4}{*}{4} & Ben 86052 & $0,14 \pm 0,01 \mathrm{a}$ & $0,13 \pm 0,01 \mathrm{a}$ & $0,14 \pm 0,01 \mathrm{~A}$ \\
\hline & Bouaké & $0,15 \pm 0,01 \mathrm{a}$ & $0,16 \pm 0,01 \mathrm{a}$ & $0,13 \pm 0,01 \mathrm{~A}$ \\
\hline & Odongbo & $0,15 \pm 0,01 \mathrm{a}$ & $0,17 \pm 0,01 \mathrm{a}$ & $0,16 \pm 0,01 \mathrm{~A}$ \\
\hline & Moyennes & $0,15 \pm 0,01 X$ & $0,15 \pm 0,01 X$ & \\
\hline
\end{tabular}

N.B. : Les moyennes suivies de la même lettre alphabétique de même caractère et pour le même facteur (les lettres $a$, $b$ montrent les différences entre les jachères manioc considérant le facteur fertilisation et sans fertilisation ; A, B montrent les différences entre les jachères manioc tous les niveaux de fertilisation confondus ; $\mathrm{X}, \mathrm{Y}$ montrent les différences entre les niveaux de fertilisation tous les types de jachère manioc confondus) ne sont pas significativement différentes $(\mathrm{P}>0,05)$ d'après le test de Student Newman-Keuls.

Effet des types de 'jachères' manioc, de la séquence des cultures et des arrières effets d'une précédente fumure minérale sur la dynamique des champignons endomycorhiziens dans le sol

Le nombre de spores de champignons endomycorhiziens présentes dans le sol a été évalué avant la première culture et après la dernière culture de maïs. Les sporocarpes et les spores ont été indifféremment pris en compte. D'une façon générale, les types de 'jachère', la précédente fumure minimale et les séquences de culture ont significativement $(\mathrm{P}<0,001)$ influencé le nombre de spores dans le sol. Entre les deux périodes d'estimation, le nombre de spores a significativement $(\mathrm{P}<0,001)$ augmenté dans les pots non fumés et diminué dans ceux ayant reçu une précédente fumure pour se stabiliser autour de 10,67 spores/g de sol sec dans les deux cas (Figure 2). Globalement, le nombre de spores dans le sol de la 'jachère' Ben 86052 avant la première culture est 1,2 et 1,9 fois significativement $(\mathrm{P}<0,001)$ supérieur à ceux des 'jachères' Bouaké et Odongbo respectivement contre 1,2 fois après la quatrième culture de maïs en pot.

Effet des types de 'jachères' manioc, de la succession des cultures et de l'arrière effet d'une fumure minérale sur le taux d'infection endomycorhizienne des racines de maïs

Les résultats du taux de colonisation des racines confirment l'hypothèse $d u$ transfert des spores de champignons endomycorhiziens des sols sous 'jachère' manioc aux cultures subséquentes de maïs. Les racines des quatre cultures successives ont été différemment colonisées par les champignons endomycorhiziens. Les types de 'jachère', l'effet de la précédente fumure et la séquence des cultures influent significativement $(\mathrm{P}<0,001)$ sur le taux de colonisation des racines par les spores des champignons endomycorhiziens. L'évolution des taux de colonisation, telle que présentée par la figure 3 ne met pas en évidence une tendance nette. Elle fluctue autour d'une valeur moyenne de $24,67 \pm 0,69 \%$. $\mathrm{La}$ 

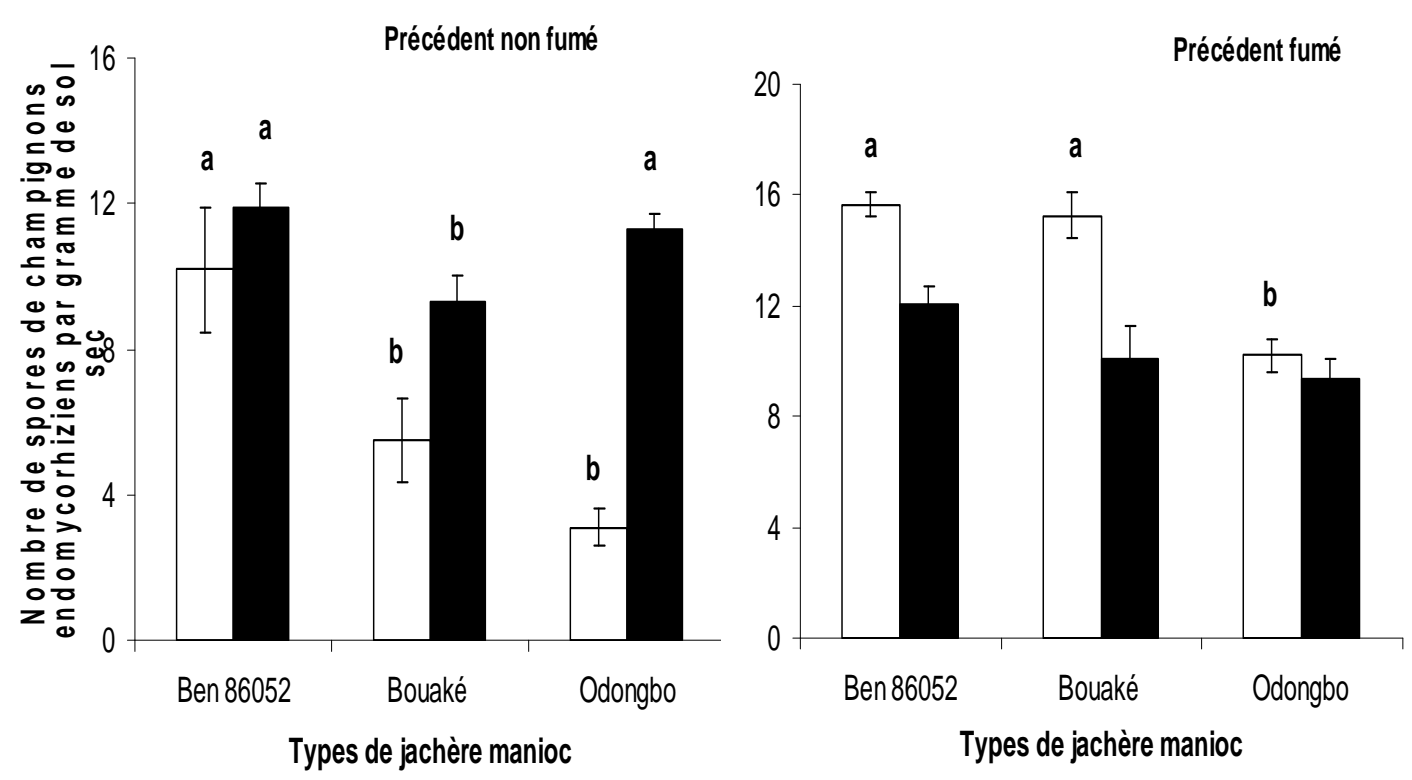

$\square$ A la récolte du manioc

- A la quatrième culture successive de maïs

Figure 2: Effets des types de 'jachère' manioc et de l'arrière effet d'une fumure de $\mathrm{N}_{14} \mathrm{P}_{23} \mathrm{~K}_{14} \mathrm{~S}_{5} \mathrm{~B}_{1}$ sur l'évolution de la densité de spores et de sporocarpes de champignon endomycorhizien au début et après la $4{ }^{\text {ème }}$ culture de maïs en pots. Les barres représentent les erreurs standard. Les barres de même couleur et portant la même lettre alphabétique ne sont pas significativement différentes selon le test de Student Newman Keuls $(\mathrm{P}>0,05)$. Ceux non étiquetés ne le sont pas non plus.

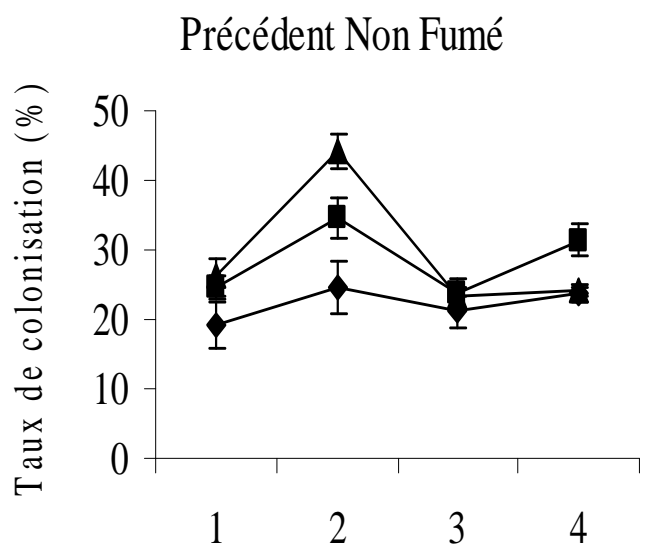

Succession des cultures

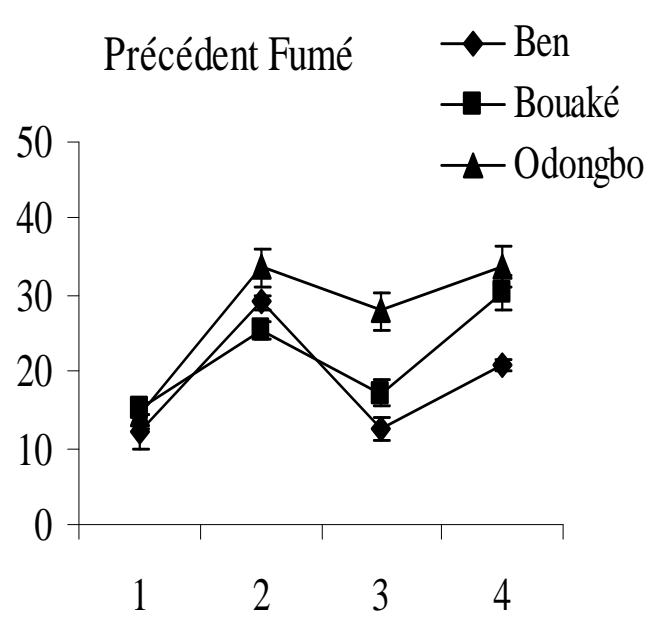

Succession des cultures

Figure 3: Effets des types de 'jachère' manioc et de l'arrière effet d'une précédente fumure minérale sur l'évolution du taux de colonisation des racines des plants de maïs en pots par les spores des champignons endomycorhiziens. Les barres verticales représentent les erreurs standard. 
colonisation des racines est significativement $(\mathrm{P}<0,001)$ plus élevée au niveau des plants de la 'jachère' Odongbo comparativement aux 'jachères' Ben 86052 et Bouaké, ceci lors des $2^{\text {ème }}, 3^{\text {ème }}$ et $4^{\text {ème }}$ cultures au niveau des précédents fumés. L'arrière effet de la fumure sur la colonisation des racines est significatif $(\mathrm{P}<0,05)$, mais sans incidence majeure sur la croissance des plants. Dans l'ensemble, les plants sans précédente fumure sont plus colonisés que les plants sur sols précédemment fertilisés. Mais au fil des périodes de culture, l'arrière effet dépressif de la fumure sur la colonisation des racines s'est atténué pour s'annuler à la $4^{\text {ème }}$ culture.

\section{Corrélation entre les facteurs}

Le Tableau 4 montre les résultats des différentes corrélations observées sur l'ensemble des plants en pots entre le taux de colonisation des racines par les champignons endomycorhiziens et les biomasses (racinaire et aérienne) et la mobilisation du P. On note des corrélations significatives $(\mathrm{P}<0,05$ à $\mathrm{P}<0,001)$ entre le taux de colonisation des racines par les spores des champignons endomycorhiziens et le ratio biomasse aérienne/biomasse racinaire, et la mobilisation $\mathrm{du}$ P. Ces observations expliquent la prépondérance et le rôle important joué par les champignons endomycorhiziens dans le maintien des rendements culturaux dans les systèmes de culture basés sur le manioc.

\section{DISCUSSION}

Dynamique des spores de mycorhizes dans le sol après plusieurs cultures de maïs subséquentes à des 'jachères' manioc
Avant l'installation de la culture de maïs en pot, la densité des spores a été évaluée dans le sol prélevé. Les échantillons observés se sont révélés très riches en spores (en moyenne 9,99 spores/g de sol sec). Cette valeur moyenne est supérieure au seuil critique de 5 spores/g de sol sec en deçà duquel on ne peut espérer une colonisation maximale des racines par les champignons (Cardoso et Kuyper, 2006). Cette densité de spores est similaire à celle trouvée par Mathimaran et al. (2007) $(8,97 \pm 1,16$ spores/g de sol) sur sol sous maïs.

L'écart observé en début d'expérimentation entre les densités de spores sur parcelles fertilisées et non fertilisées pourrait être lié au stress subi par les mycorhizes au champ, ce qui a induit un accroissement de cette forme de résistance sur les parcelles fertilisées (plus stressantes). Baum et al. (2002) ont observé également une augmentation et une diminution de la densité de spores due à l'application de la fumure azotée, respectivement sur sols à faible et forte concentration initiale d'azote. Soumises aux mêmes conditions (stress imposé par la fertilisation), les spores ont connu une diminution de la variabilité de leur densité. Toutefois, certains facteurs intrinsèques au développement des mycorhizes comme la saisonnalité ou la production et la libération des spores par les racines infectées ne doivent pas être négligés (Cardoso et Kuyper, 2006) dans l'explication de ces variations au niveau des résultats.

Etèka (2005) avait trouvé sur les pots non fumés des corrélations significatives $(\mathrm{P}<0,05)$ entre le taux de colonisation

Tableau 4: Coefficients de corrélations de Pearson entre le taux de colonisation des racines de maïs par les champignons endomycorhiziens au cours des quatre successions culturales et la biomasse aérienne, biomasse racinaire, et la mobilisation du $\mathrm{P}$.

\begin{tabular}{|c|c|c|c|}
\hline Variables & Précédent non fumé & Précédent fumé & $\begin{array}{c}\text { Ensemble fumé et } \\
\text { non fumé }\end{array}$ \\
\hline $\begin{array}{l}\text { Biomasse aérienne } \\
\text { (g MS/plant) }\end{array}$ & $0,07 \mathrm{~ns}$ & $-0,16 \mathrm{~ns}$ & $-0,09 \mathrm{~ns}$ \\
\hline $\begin{array}{l}\text { Biomasse racinaire } \\
\text { (g MS/plant) }\end{array}$ & $-0,15 \mathrm{~ns}$ & $-0,46 * * *$ & $-0,31 * *$ \\
\hline $\begin{array}{l}\text { Biomasse aérienne / biomasse } \\
\text { racinaire }\end{array}$ & $0,21 *$ & $0,47 * * *$ & $0,32 * *$ \\
\hline $\begin{array}{l}\text { Mobilisation de } \mathrm{P} \\
\text { (mg P/plant) }\end{array}$ & $0,27 * *$ & $0,45 * * *$ & $0,35 * * *$ \\
\hline
\end{tabular}


racinaire et la biomasse aérienne, les quantités de $\mathrm{P}$ et de $\mathrm{K}$ mobilisées par la plante. L'auteur a suggéré que la présence de l'engrais inhibe la mobilisation des nutriments effectuée par les mycorhizes. Au cours de la présente étude, ce sont plutôt les plants développés sur les sols précédemment fertilisés qui ont le mieux répondu à la colonisation par les champignons endomycorhiziens. Contrairement aux observations faites par Miller (2000), cette réponse s'est exprimée non pas par le nombre de spores noires, mais par celui de l'ensemble des autres spores comme mentionné par Hougni (2005). Entre les observations faites après la 'jachère' manioc et celles de la présente étude, la proportion de spores noires aurait vraisemblablement augmenté. L'augmentation du pourcentage de spores noires serait éventuellement un élément caractéristique de la dynamique des spores de champignons indigènes dans les sols ayant porté la succession culturale manioc-maïsmaïs. Au-delà de la transmission des mycorhizes évoquée par Etèka (2005), nous émettons l'hypothèse selon laquelle le manioc, par sa présence, ait modifié la composition de la flore fongique du sol, en augmentant la densité des spores de champignons bénéfiques au maïs.

Sur les sols précédemment fertilisés, une corrélation hautement significative et négative (coefficient de corrélation de Pearson $=-0,50 ; \quad \mathrm{P}<0,001)$ est observée entre l'abondance des spores dans le sol et le taux de colonisation des racines du maïs en pots. Ce qui n'est pas le cas au niveau des sols non fertilisés (coefficient de corrélation de Pearson $=0,18 ; \mathrm{P}>0,05)$. Ces résultats dénotent que le déterminisme de la colonisation racinaire ne se restreint pas uniquement à l'abondance des spores dans le sol, mais prendrait en compte d'autres facteurs tels que les sources de P disponible pour les mycorhizes comme mentionné également par Allen et al. (2003).

\section{Implication des champignons endomyco- rhiziens dans la production de biomasse du maïs subséquent à la 'jachère' manioc}

$\mathrm{Au}$ cours de la présente étude, on a observé que les plants développés sur sols précédemment fertilisés ont le mieux répondu à la colonisation par les champignons endomycorhiziens. Contrairement aux observations faites par Ryan et Angus (2003),
Solaiman et Abbott (2003), cette réponse s'est exprimée, non pas par l'accroissement des teneurs en éléments nutritifs, mais par l'augmentation de la biomasse aérienne. La corrélation avec les éléments nutritifs mobilisés est moins marquée que sur les plants de la précédente culture de maïs.

$\mathrm{Au}$ sein des pots non fumés, l'augmentation du niveau de colonisation racinaire n'a pas entraîné une amélioration de la nutrition de la plante-hôte. Dans le cas spécifique du $\mathrm{P}$, une telle situation a été également rapportée par Mathimaran et al. (2007) sur sol ferrallitique au Kenya. De plus, Ryan et Angus (2003), ont observé sur le blé que l'augmentation du taux de colonisation n'induit pas ipso facto une amélioration de la production des plantes ni de la concentration du phosphore dans la plante. Des résultats similaires ont été observés par Ryan et Graham (2002) étudiant le rôle potentiel des champignons mycorhiziens dans la production agricole. Ces auteurs ont mentionné que, quand bien même la disponibilité du $\mathrm{P}$ est faible et le niveau de colonisation élevé, les champignons endomycorhiziens, pour des raisons non encore élucidées peuvent ne pas contribuer à la croissance des plantes-hôtes. L'ineffectivité de la symbiose expliquerait bien par ailleurs, l'absence de réponse de la plante-hôte à la colonisation racinaire observée dans le cas de la présente étude et qui a été également rapportée par Kelly et al. (2001) et Ryan et Angus (2003).

$\mathrm{Au}$ niveau des pots précédemment fertilisés, on remarque à chaque culture, excepté la deuxième (dont le taux de colonisation racinaire est le plus élevé), que le taux de colonisation racinaire est significativement $(\mathrm{P}<0,01)$ corrélé avec la production de biomasse aérienne. Comparativement aux plants des pots non fertilisés, les coefficients de corrélation y sont plus élevés et généralement plus significatifs. L'effectivité de la réponse pourrait être attribuée à l'absorption dans le sol du $\mathrm{P}$ apporté par l'engrais. Il est évident que les quantités relativement élevées de $\mathrm{P}$ assimilable n'aient pas "motivé" le développement mycorhizien, comme ce fut le cas dans l'étude réalisée par Graham et Abots (2000) lorsqu'une application minérale de P était faite. Mais, avec le temps, l'effet inhibiteur a dû s'estomper. Par ailleurs, le 
constat fait à la deuxième culture confirmerait l'hypothèse de Miller (2000) selon laquelle l'accroissement du taux de colonisation audelà d'un certain seuil n'entraîne pas une amélioration de la nutrition phosphatée et du rendement de la plante-hôte. Aussi, faut-il noter sur ces mêmes plants que la corrélation avec la mobilisation du $\mathrm{P}$ a diminué au fil de l'expérimentation. Ceci ne serait que la conséquence de l'amenuisement progressif du $\mathrm{P}$ mobilisable par les mycorhizes comme mentionné également par Jansa et al. (2005). Nous émettons l'hypothèse que la poursuite des cultures de maïs sur ces sols conduirait à une situation similaire à celle observée actuellement sur les pots non fertilisés mais ceci doit être confirmé par d'autres investigations au champ.

\section{Conclusion}

Les résultats du taux de colonisation des racines confirment l'hypothèse du transfert des spores de champignons endomycorhiziens des sols sous 'jachère' manioc aux cultures subséquentes de maïs. Les résultats dénotent également le rôle important joué par les champignons endomycorhiziens dans le maintien des rendements culturaux dans les systèmes de culture basés sur le manioc. Toutefois, dans une culture successive de maïs, les plants sont différemment colonisées par les champignons endomycorhiziens. La fumure minérale spécialement l'apport de $\mathrm{P}$ exerce un arrière effet dépressif sur la colonisation des racines par les champignons mycorhiziens. Mais, au fil du temps, cet arrière effet s'atténue. L'efficacité des mycorhizes dans l'amélioration de la mobilisation du $\mathrm{P}$ est donc confirmée et semble être sous le contrôle de l'apport du P. La colonisation des racines par les spores des champignons endomycorhiziens a contribué à l'amélioration de la mobilisation du phosphore. Cependant, l'efficacité de leur action chute considérablement au cours des cultures successives, probablement à cause de l'épuisement du sol en phosphore.

\section{REFERENCES BIBLIOGRAPHIQUES}

Adjei-Nsah S, Kuyper TW, Leeuwis C, Abekoe MK, Giller KE. 2007. Evaluating sustainable and profitable cropping sequences with cassava and four legume crops: effects on soil fertility and maize yields in the forest/savannah transitional agro-ecological zone of Ghana. Field Crop Res., 103: 87-97.

Allen MF, Swenson W, Querejeta JI, Egerton Warburton LM, Treseder KK. 2003. Ecology of mycorrhizae: a conceptual framework for complex interactions among plants and fungi. Annual Review of Phyto., 41: 271-303.

Baum C, Weih M, Verwijst T, Makeschin F. 2002. The effects of nitrogen fertilization and soil properties on mycorrhizal formation of Salix viminalis. For. Ecol. Man., 160: 35-43.

Brundrett M, Bougher N, Dell B, Grove T, Malajczuk N. 1996. Working with mycorrhizas in forestry and agriculture. Australian Centre for International Agricultural Research, Monograph 32, Canberra, Australia, 374.

Cardoso IM, Kuyper TW. 2006. Mycorrhizas and tropical soil fertility. Agric. Ecosyst. Enviro., 116: 72-84.

Dagnelie P. 1998. Statistique théorique et appliquée (Tome 2). In Inférences Statistiques à Une et Deux Dimensions, Boeck, Larcier (eds). Paris-Bruxelles: France, Belgique; 659.

Douthwaite B, Manyong VM, Keatinge JDH, Chiau J. 2002. The adoption of alley farming and mucuna: lessons for research, development and extension. Agrof. Syst., 56: 193-202.

Erenstein O. 2003. Smallholder conservation farming in the tropics and sub-tropics: a guide to the development and dissemination of mulching with crop residues and cover crops. Agric. Ecosyst. Environ., 100: 17-37.

Etèka AC. 2005. Contribution des 'jachères' manioc dans l'amélioration du rendement des cultures et du prélèvement des nutriments : cas de la succession culturale manioc-maïs au centre du Bénin. Thèse de DEA, FSA/UAC, Abomey-Calavi, Bénin, 107.

Fagbola O, Osonubi O, Mulongoy K. 1998. Contribution of arbuscular mycorrhizal (AM) fungi and hedgerow trees to the yield and nutrient uptake of cassava in alley-cropping system. J. Agric. Sci., Cambridge, 131: 79-85.

Fermont AM, van Asten PJA, Giller KE. 2008. Increasing land pressure in East 
Africa: the changing role of cassava and consequences for sustainability of farming systems. Agric. Ecosyst. Environ., 128: 239-250.

Giovannetti M, Mosse B. 1980. An evaluation of techniques for measuring vesicular arbuscular mycorrhizal infection in roots. New Phytol., 84: 489-500.

Graham JH, Abbott LK. 2000. Wheat responses to aggressive and nonaggressive arbuscular mycorrhizal fungi. Plant Soil, 220: 207-218.

Habte M, Byappanahalli MN. 1994. Dependency of cassava (Manihot esculenta Crantz) on vesicular-arbuscular mycorrhizal fungi. Mycorrhiza, 4: 241245.

Hougni JMD-G. 2005. Durabilité des jachères manioc dans le maintien de la fertilité des sols au Centre Bénin: contribution des champignons mycorhiziens à la mobilisation de l'azote et du phosphore. Thèse Ingénieur Agronome, FSA Université d'Abomey-Calavi, AbomeyCalavi, Bénin, 121.

Howeler RH. 2002. Cassava mineral nutrition and fertilization. In Cassava: Biology, Production and Utilisation, Hillocks RJ, Thresh JM, Bellotti AC (eds). CAB International: Cali, Colombia; 115-147.

Jansa J, Mozafar A, Frossard E. 2005. Phosphorus acquisition strategies within arbuscular mycorrhizal fungal community of a single field site. Plant Soil, 276: 163-176.

Kelly RM, Edwards DG, Thompson JP, Magarey RC. 2001. Responses of sugarcane, maize, and soybean to phosphorus and vesicular-arbuscular mycorrhizal fungi. Aust. J. Agric. Res., 52: 731-743.

Kolawolé GO, Tian G, Tijani-Eniola H. 2003. Dynamics of phosphorus fractions during fallow with natural vegetation and planted Pueraria phaseoloides in southwestern Nigeria. Plant Soil, 257: 63-70.

Mathimaran N, Ruh R, Jama B, Verchot L, Frossard E, Jansa J. 2007. Impact of agricultural management on arbuscular mycorrhizal fungal communities in
Kenyan ferralsol. Agric. Ecosyst. Environ., 119: 22-32.

Miller MH. 2000. Arbuscular mycorrhizae and phosphorus nutrition of maize: a review of Guelph studies. Can. J. Plant Sci., 80: 47-52.

Ryan MH, Angus JF. 2003. Arbuscular mycorrhizae in wheat and field pea crops on a low P soil: increased Zn-uptake but no increase in P-uptake or yield. Plant Soil, 250: 225-239.

Ryan MH, Graham JH. 2002. Is there a role for arbuscular mycorrhizal fungi in production Agriculture ? Plant Soil, 244: 263-271.

Saïdou A, Kuyper TW, Kossou DK, Tossou R, Richards P. 2004. Sustainable soil fertility management in Bénin: learning from farmers. NJAS - Wag. J. Life Sci., 52: 349-369.

Saïdou A. 2006. Converging strategies by farmers and scientists to improve soil fertility and enhance crop production in Benin. $\mathrm{PhD}$ thesis, Wageningen University, The Netherlands, 223.

Saïdou A, Kossou D, Brussaard L, Richards P, Kuyper TW. 2008. Earthworm activities in cassava and egusi melon fields in the transitional zone of Benin: linking farmers' perceptions with field studies. NJAS - Wag. J. Life Sci., 56(1\&2): 123135.

Sanchez PA, Jana BA. 2002. Soil fertility replenishment takes off in East and Southern Africa. In Intagrated Plant Nutrient Management in Sub-Saharan Africa: from Concept to Practice, Vanlauwe B, Diels J, Sanginga N, Merckx (eds). CAB International: Wallingford, UK; 23-45.

Serpantié G, Ouattara B. 2001. Fertilité et jachères en Afrique de l'Ouest. In $L a$ Jachère en Afrique Tropicale (Vol 2), Floret Ch, Pontanier R (eds). John Libbey Eurotext: Paris; 21-83.

Solaiman MZ, Abbott LK. 2003. Phosphorus uptake by a community of arbuscular mycorrhizal fungi in jarrah forest. Plant Soil, 248: 313-320. 